\title{
Article \\ An Assessment of the Nutritional Value of the Preschool Food Rations for Children from the Wroclaw District, Poland-The Case of a Big City
}

\author{
Agnieszka Orkusz
}

check for updates

Citation: Orkusz, A. An Assessment of the Nutritional Value of the Preschool Food Rations for Children from the Wroclaw District, Poland-The Case of a Big City. Nutrients 2022, 14, 460. https:// doi.org/10.3390/nu14030460

Academic Editor: Iain A. Brownlee

Received: 10 December 2021

Accepted: 19 January 2022

Published: 20 January 2022

Publisher's Note: MDPI stays neutral with regard to jurisdictional claims in published maps and institutional affiliations.

Copyright: (C) 2022 by the author. Licensee MDPI, Basel, Switzerland. This article is an open access article distributed under the terms and conditions of the Creative Commons Attribution (CC BY) license (https:// creativecommons.org/licenses/by/ $4.0 /)$.
Department of Biotechnology and Food Analysis, Wroclaw University of Economics and Business, 53-345 Wroclaw, Poland; agnieszka.orkusz@ue.wroc.pl; Tel.: +48-713680480

\begin{abstract}
The evaluation of nutrition is an essential element of preventing chronic diseases and can be used to determine nutritional recommendations. A child spends about $7-8 \mathrm{~h}$ a day in a kindergarten; therefore, meals served there should be balanced appropriately to ensure the full psychophysical development of the young organism. At preschool age, children develop eating habits that can have life-long effects. Based on 10-day menus, the study aimed to estimate the energy and nutritional value of children's diets at four randomly selected kindergartens in the Wroclaw district, Poland. In total, 80 menus were analyzed (40 for summer and 40 for autumn). The data from kindergartens were analyzed based on the Diet 6D computer program. Regardless of the kindergarten, the analyzed food rations showed irregularities related to excessive supplies (in reference to the dietary recommendations) of sucrose, fiber, salt, magnesium, and vitamin A. The preschool food rations did not cover demands with respect to PUFA n-3, PUFA-n-6, calcium, and vitamin D. The observed irregularities confirm the need to monitor the content of energy and nutrients in preschool menus to be able to correct any dietary errors.
\end{abstract}

Keywords: child; diet; kindergarten; menus; nutritional errors

\section{Introduction}

The proper nutrition of pre-school children guarantees their optimal health $[1,2]$. Because a child spends about 7-8 h a day in kindergarten, the food served there should be balanced appropriately to provide energy and nutrients in the right amounts and proportions [3]. The collective nutrition of children and adolescents within the education system must meet the appropriate requirements for given age groups, adhering to the current nutrition standards for the Polish population [4]. The following standards were adopted in the latest 2020 Polish nutrition standards: Estimated Energy Requirement (EER); Estimated Average Requirement (EAR); Recommended Dietary Allowance (RDA); Adequate Intake (AI), and Reference Intake ranges for macronutrients (RIs) [4]. In planning mass nutrition in kindergartens, the Recommended Dietary Allowance (RDA) level should be applied. This guarantees that the planned nutrition will cover the average demand of the group of preschoolers at the EAR level [5]. In addition, the Tolerable Upper Intake Level (UL) has been developed for vitamins and mineral ingredients. The UL is the maximum level of nutrient intake from all sources (food, drinking water, dietary supplements) that does not cause adverse health effects in almost all people in each group. Consumption of nutrients above this level may cause adverse health effects. The UL value is a guideline level that should not be pursued as a standard but should be observed to ensure that it is not exceeded [4]. A child receives at least 3 meals daily in kindergarten. Therefore, it is assumed that the daily preschool ration should cover $75 \%$ of energy and nutrient needs [2,5-10].

Proper nutrition in kindergarten is also crucial for shaping the correct eating habits of children and can prevent the later development of many diet-related diseases (obesity, type 
2 diabetes, cardiovascular diseases) [11]. The statistics related to obesity among children and adolescents are worrying across the world, including Poland. Data from the World Health Organization indicate an upward trend in obesity among children aged 5-9 and $10-19$ [12]. Over 10 years, boys showed an increase in obesity of $6.3 \%$ and $4.8 \%$, respectively, while girls showed an increase of $3.0 \%$ and $1.7 \%$. It is estimated that, by 2025 , if the rate of increase in obesity continues to grow at a similar pace, about 268 million children aged 5-17 years will be overweight, of whom 91 million will be obese [13]. In 2025, 38 million children will have hepatic steatosis or a buildup of fat in the liver, 27 million will have hypertension, 12 million will suffer from impaired glucose tolerance, and 4 million will have type 2 diabetes [14]. A lot of studies show that obesity in childhood is strongly associated with obesity in adulthood [15-18]. Most children who gained excess weight before the age of 6 remain overweight during puberty [18]. It has been shown that the earlier the increase in body fat occurs, the greater the final weight of the child is $[15,19]$.

Many authors point to improper nutrition of children in kindergartens in Poland [7,20-31]. Meals are improperly balanced, both in terms of energy and nutritional value. It also happens that they are prepared from low-quality ingredients. This situation may be due to the insufficient knowledge and skills of the people responsible for preschool nutrition, which suggests the need to implement nutritional education for staff [2]. Perhaps a regular assessment of menus would help to avoid basic mistakes that occur when composing them.

Based on 10-day menus, the study aimed at estimating the energy and nutritional value of children's diets in four randomly selected kindergartens in the Wroclaw district, Poland.

\section{Materials and Methods}

\subsection{Data}

The research was conducted in 2019. The menus from the 4 randomly selected preschool institutions (designated as: A, B, C, D) located in the Wroclaw district were assessed. There were 5-7 pre-school groups in the facilities, to which children aged 4 to 6 were assigned. Each group consisted of about 25 children. The kindergartens had their own kitchens where meals were prepared (the services of catering companies were not used). The 10-day menus from the summer and autumn seasons were assessed in each of 4 kindergartens. In total, 80 menus were analyzed (40 menus for summer and 40 menus for autumn).

To assess the energy value and the content of nutrients in the food rations, such as proteins, fats, carbohydrates (including glucose, fructose, sucrose, lactose, starch), fiber, minerals (sodium $(\mathrm{Na})$, potassium $(\mathrm{K})$, calcium $(\mathrm{Ca})$, phosphorus $(\mathrm{P})$, magnesium $(\mathrm{Mg})$, iron $(\mathrm{Fe})$, zinc $(\mathrm{Zn})$, copper $(\mathrm{Cu})$, and manganese $(\mathrm{Mn})$ ), vitamins (thiamine $\left(\mathrm{B}_{1}\right)$, riboflavin $\left(B_{2}\right)$, niacin $\left(B_{3}\right)$, pyridoxine $\left(B_{6}\right)$, and vitamins $A, C, D$, and $\left.E\right)$, cholesterol, and fatty acids (saturated, monounsaturated, polyunsaturated), the Diet 6D computer program [32] was implemented. The salt content was also calculated. The Diet 6D program has proved to be the best in Poland so far. It offers the possibility of calculating energy intake and as many as 89 nutrients. The Diet program contains a food composition database based on the Polish food composition tables. The portion size was estimated based on the number of products, meals, and drinks expressed in grams specified in the menus. The calculations considered losses resulting from technological processes. Therefore, for the energy value, total protein, fats, carbohydrates, calcium, and iron, losses equal to $10 \%$ were used, and for vitamins $\mathrm{A}$ and C, losses equivalent to $20 \%$ and $55 \%$, respectively, were used [33]. Then all values for 10-day menus were totaled. In this way, for each kindergarten, the average values of energy and nutrients were estimated. Next, the obtained results were compared with the applicable standards and recommendations for children aged 4-6 years [4]. Energy standards were set at the level of average demand (Estimated Energy Requirement). The Reference Intake ranges, expressed as a percentage of the energy requirement, were defined for fat and carbohydrates. The standards for protein, selected minerals (calcium, phosphorus, magnesium, iron, zinc, copper), and vitamins (thiamine, riboflavin, niacin, pyridoxine, vitamins A and C) were set as per the Recommended Dietary 
Allowance. Standards for other nutrients (sodium, potassium, manganese, vitamins D and E, fiber, n-3 PUFA, and n-6 PUFA) were set as per the level of sufficient consumption (Adequate Intake) [4].

To assess the diet of children aged 4-6 years, with a bodyweight of $19 \mathrm{~kg}$ and moderate physical activity, a daily energy requirement of $1400 \mathrm{kcal}$ was adopted in accordance with the standards [4]. For children up to the age of 9, the standards are the same for boys and girls. Their differentiation by gender is applied in the older age groups. The energy standards for children up to 6 years of age include the same levels of physical activity. The norms for energy, protein, and fat were established based on body weight, which is understood as a reference body weight value, not the actual weight of a child. The data from studies representative of the Polish population were used to determine the reference body weight values for preschool children (3-6 years) [34]. The reference body weight value for children aged 4-6 years, based on the median, is $19 \mathrm{~kg}$. The energy and nutrient content of the preschool diet was compared to $75 \%$ of the daily norm for children aged 4-6 [2,5-10]. According to this, the recommended value for energy was $1050 \mathrm{kcal}$ [4]. The consumption values differing by $\pm 10 \%$ from the standard values were assumed to be correct. The content of minerals $(\mathrm{Mg}, \mathrm{Zn}, \mathrm{Cu})$ and vitamins $\left(\mathrm{A}, \mathrm{B}_{6}\right)$ was also compared to 75\% of the Tolerable Upper Intake Level [35]. Various groups of experts have established UL levels for individual vitamins and minerals. UL values proposed by the European Food Safety Authority were used in the study [35]. It was assumed that the children consumed all served food.

\subsection{Statistical Analysis}

Two-way variance analysis (ANOVA) was used to evaluate changes in energy value and nutrients in kindergarten menus. The effect of such factors as the kindergarten (A, B, C, D), the season (summer and autumn), and a couple of interactions between them were analyzed. When a significant main effect or interaction was identified, the mean values were further analyzed using the Tukey's multiple range test. The results were presented as a mean \pm standard deviation. The experimental data were analyzed using Statistica version 13.3 (StatSoft Inc., Kraków, Poland) [36]. Differences with a probability level $<0.05$ were considered significant.

\subsection{Ethical Statement}

Ethical review and approval were waived for this study as the research was based on an evaluation of the preschool menus based on the computer program Diet 6D.

\section{Results}

Based on the analysis of variance results, it was shown that for energy and nutrients (except for copper, manganese, cholesterol, and PUFA n-3), the main effect was significant for the type of kindergarten for the adopted significance level of $p<0.05$. For most nutrients, the season factor was not found to be significant. A two-way interaction effect occurred only with carbohydrates and starch (Table 1).

Based on the results of this research, it was shown that the energy value of meals differed significantly depending on the kindergarten, both in summer and in autumn (Table 1). The energy value of meals served in summer was correct in kindergartens A, $\mathrm{B}$ and $\mathrm{C}$, while in autumn, this was the case only in kindergartens A and B. The energy value of menus in the kindergarten D was higher than the Estimated Energy Requirement, regardless of the season (Table 2). 
Table 1. Mean values ( \pm standard deviation) of energy value and nutrient content in preschool menus.

\begin{tabular}{|c|c|c|c|c|c|c|c|c|}
\hline \multirow{2}{*}{$\begin{array}{c}\text { Energy } \\
\text { and Nutrients }\end{array}$} & \multirow{2}{*}{ Season (S) } & \multicolumn{4}{|c|}{ Kindergarten (K) } & \multicolumn{3}{|c|}{ Significant Effects } \\
\hline & & A & B & $\mathrm{C}$ & D & $\mathbf{K}$ & $\mathrm{S}$ & $\mathbf{K} \times \mathbf{S}$ \\
\hline \multirow[t]{2}{*}{ Energy (kcal) } & Summer & $945.4^{\mathrm{a}} \pm 205.7$ & $1024.4^{\mathrm{a}} \pm 148.9$ & $1059.8^{\mathrm{a}} \pm 138.3$ & $1397.3^{b} \pm 13.5$ & $* * *$ & $* *$ & ns \\
\hline & Autumn & $1121.8^{\mathrm{a}} \pm 116.7$ & $1029.4^{\mathrm{a}} \pm 108.5$ & $1217.7^{\mathrm{a}} \pm 238.7$ & $1397.1^{b} \pm 6.6$ & & & \\
\hline \multirow[t]{2}{*}{ Protein (g) } & Summer & $30.7^{a} \pm 6.7$ & $35.8^{a} \pm 3.6$ & $36.2^{\mathrm{a}} \pm 8.0$ & $52.3^{b} \pm 9.6$ & $* * *$ & * & ns \\
\hline & Autumn & $40.5^{a} \pm 9.6$ & $37.5^{\mathrm{a}} \pm 4.8$ & $39.1^{\mathrm{a}} \pm 10.0$ & $55.4^{\mathrm{b}} \pm 14.4$ & & & \\
\hline \multirow[t]{2}{*}{ Fat $(\mathrm{g})$} & Summer & $29.4^{\mathrm{ab}} \pm 13.3$ & $24.6^{\mathrm{a}} \pm 4.4$ & $23.6^{\mathrm{a}} \pm 4.5$ & $40.7^{b} \pm 14.5$ & $* * *$ & ns & ns \\
\hline & Autumn & $30.1^{\mathrm{ab}} \pm 7.5$ & $29.6^{\mathrm{ab}} \pm 7.5$ & $22.8^{\mathrm{a}} \pm 7.8$ & $42.8^{b} \pm 12.9$ & & & \\
\hline \multirow[t]{2}{*}{ Carbohydrates (g) } & Summer & $146.7^{\mathrm{a}} \pm 27.3$ & $171.5^{\mathrm{ab}} \pm 33.5$ & $183.7^{a b} \pm 29.8$ & $212.6^{\mathrm{b}} \pm 33.1$ & $* * *$ & ns & * \\
\hline & Autumn & $181.0^{\mathrm{ab}} \pm 26.7$ & $159.4^{\mathrm{a}} \pm 24.4$ & $222.2^{b} \pm 54.8$ & $205.8^{a b} \pm 28.4$ & & & \\
\hline \multirow[t]{2}{*}{ Sodium (mg) } & Summer & $1763.3^{a} \pm 332.5$ & $1747.5^{\mathrm{a}} \pm 382.2$ & $2100.5^{a} \pm 422.6$ & $2261.3^{a} \pm 755.9$ & $* *$ & ns & ns \\
\hline & Autumn & $2172.6^{a b} \pm 386.7$ & $1756.5^{\mathrm{a}} \pm 282.0$ & $1974.3^{a b} \pm 378.0$ & $2448.2^{b} \pm 574.5$ & & & \\
\hline \multirow[t]{2}{*}{ Potassium (mg) } & Summer & $1928.1 \pm 374.5$ & $1652.0 \pm 350.7$ & $2085.4 \pm 455.0$ & $2070.4 \pm 589.4$ & $* *$ & ns & ns \\
\hline & Autumn & $2337.7^{a} \pm 550.3$ & $1577.2^{b} \pm 321.7$ & $1988.1^{\mathrm{ab}} \pm 400.0$ & $2313.3^{\mathrm{ab}} \pm 535.5$ & & & \\
\hline \multirow[t]{2}{*}{ Calcium (mg) } & Summer & $250.4^{\mathrm{a}} \pm 102.6$ & $401.9^{\mathrm{ab}} \pm 130.3$ & $373.2^{\mathrm{ab}} \pm 107.4$ & $473.2^{b} \pm 201.4$ & $* * *$ & ns & ns \\
\hline & Autumn & $364.0^{\mathrm{a}} \pm 148.9$ & $380.6^{a} \pm 110.2$ & $365.9^{a} \pm 111.3$ & $622.3^{b} \pm 222.8$ & & & \\
\hline \multirow{2}{*}{ Phosphorus (mg) } & Summer & $476.6^{\mathrm{a}} \pm 121.3$ & $652.8^{\mathrm{ab}} \pm 82.2$ & $653.0^{\mathrm{ab}} \pm 163.5$ & $821.2^{b} \pm 171.1$ & $* * *$ & $* *$ & ns \\
\hline & Autumn & $643.8^{\mathrm{a}} \pm 191.7$ & $679.4^{\mathrm{a}} \pm 141.3$ & $675.3^{a} \pm 123.8$ & $1022.6^{b} \pm 257.2$ & & & \\
\hline \multirow[t]{2}{*}{ Calcium/Phosphorus } & Summer & $0.5 \pm 0.2$ & $0.6 \pm 0.2$ & $0.6 \pm 0.1$ & $0.6 \pm 0.1$ & ns & ns & ns \\
\hline & Autumn & $0.6 \pm 0.1$ & $0.6 \pm 0.1$ & $0.5 \pm 0.1$ & $0.6 \pm 0.2$ & & & \\
\hline Magnesium (mg) & Summer & $142.0 \pm 35.3$ & $170.4 \pm 30.0$ & $182.2 \pm 46.5$ & $196.8 \pm 51.0$ & $* *$ & * & ns \\
\hline & Autumn & $194.9 \pm 49.7$ & $188.9 \pm 57.3$ & $179.5 \pm 37.5$ & $259.5 \pm 113.8$ & & & \\
\hline Iron (mg) & Summer & $5.3 \pm 0.7$ & $5.9 \pm 0.8$ & $6.2 \pm 1.5$ & $7.3 \pm 1.3$ & $*$ & * & ns \\
\hline & Autumn & $7.0^{\mathrm{ab}} \pm 1.8$ & $6.2^{\mathrm{a}} \pm 1.4$ & $6.5^{\mathrm{a}} \pm 1.1$ & $9.8^{b} \pm 1.7$ & & & \\
\hline Zinc (mg) & Summer & $3.9^{a} \pm 0.6$ & $5.1^{\mathrm{ab}} \pm 0.5$ & $4.7^{\mathrm{ab}} \pm 1.1$ & $6.1^{b} \pm 1.7$ & $* * *$ & * & ns \\
\hline & Autumn & $5.4 \pm 1.9$ & $5.4 \pm 1.3$ & $5.0 \pm 1.0$ & $6.8 \pm 1.7$ & & & \\
\hline Copper (mg) & Summer & $0.7 \pm 0.1$ & $0.9 \pm 0.2$ & $0.8 \pm 0.2$ & $0.9 \pm 0.3$ & ns & ns & ns \\
\hline & Autumn & $0.9 \pm 0.2$ & $0.9 \pm 0.2$ & $0.8 \pm 0.1$ & $1.0 \pm 0.3$ & & & \\
\hline Manganese (mg) & Summer & $2.3 \pm 0.3$ & $2.7 \pm 0.6$ & $2.5 \pm 0.8$ & $2.8 \pm 1.2$ & ns & ns & ns \\
\hline & Autumn & $2.8 \pm 1.4$ & $3.0 \pm 0.4$ & $2.5 \pm 0.5$ & $3.5 \pm 1.7$ & & & \\
\hline Vitamin $\mathrm{B}_{1}(\mathrm{mg})$ & Summer & $0.6 \pm 0.2$ & $0.6 \pm 0.2$ & $0.5 \pm 0.2$ & $0.8 \pm 0.3$ & * & ns & ns \\
\hline & Autumn & $0.8 \pm 0.3$ & $0.6 \pm 0.1$ & $0.7 \pm 0.2$ & $0.7 \pm 0.3$ & & & \\
\hline Vitamin $B_{2}(\mathrm{mg})$ & Summer & $0.6^{\mathrm{a}} \pm 0.1$ & $0.9^{\mathrm{ab}} \pm 0.3$ & $0.8^{\mathrm{ab}} \pm 0.2$ & $1.0^{\mathrm{b}} \pm 0.2$ & $* * *$ & * & ns \\
\hline & Autumn & $0.9^{\mathrm{a}} \pm 0.3$ & $0.9^{\mathrm{ab}} \pm 0.1$ & $0.8^{\mathrm{a}} \pm 0.3$ & $1.2^{\mathrm{b}} \pm 0.3$ & & & \\
\hline Niacin (mg) & Summer & $7.8 \pm 3.3$ & $5.4 \pm 1.6$ & $8.2 \pm 3.8$ & $10.4 \pm 3.6$ & $* * *$ & ns & ns \\
\hline & Autumn & $9.7^{a b} \pm 2.5$ & $5.4^{\mathrm{a}} \pm 1.6$ & $8.4^{\mathrm{ab}} \pm 2.9$ & $12.0^{\mathrm{b}} \pm 8.5$ & & & \\
\hline Vitamin C (mg) & Summer & $77.5 \pm 27.6$ & $51.9 \pm 12.6$ & $67.0 \pm 29.8$ & $48.2 \pm 20.6$ & $* * *$ & ns & ns \\
\hline & Autumn & $98.1^{\mathrm{a}} \pm 70.0$ & $37.4^{b} \pm 16.4$ & $62.0^{\mathrm{ab}} \pm 22.9$ & $55.0^{\mathrm{ab}} \pm 22.6$ & & & \\
\hline Vitamin A $(\mu \mathrm{g})$ & Summer & $857.4 \pm 309.8$ & $689.6 \pm 281.1$ & $758.9 \pm 426.2$ & $827.9 \pm 277.4$ & * & ns & ns \\
\hline & Autumn & $975.3^{a b} \pm 370.7$ & $631.2^{\mathrm{a}} \pm 346.1$ & $801.6^{\mathrm{ab}} \pm 289.0$ & $1210.3^{b} \pm 702.4$ & & & \\
\hline Vitamin E (mg) & Summer & $4.0 \pm 1.0$ & $3.7 \pm 0.7$ & $3.4 \pm 0.7$ & $4.7 \pm 1.0$ & $* * *$ & ns & ns \\
\hline & Autumn & $4.0^{\mathrm{a}} \pm 0.7$ & $4.3^{\mathrm{ab}} \pm 1.6$ & $3.7^{\mathrm{a}} \pm 1.1$ & $5.5^{\mathrm{b}} \pm 1.5$ & & & \\
\hline Vitamin $\mathrm{B}_{6}(\mathrm{mg})$ & Summer & $1.0 \pm 0.2$ & $0.8 \pm 0.2$ & $1.1 \pm 0.3$ & $1.2 \pm 0.4$ & $* * *$ & ns & ns \\
\hline & Autumn & $1.2^{\mathrm{a}} \pm 0.2$ & $0.8^{\mathrm{b}} \pm 0.3$ & $1.0^{\mathrm{ab}} \pm 0.3$ & $1.2^{\mathrm{a}} \pm 0.5$ & & & \\
\hline Vitamin D $(\mu \mathrm{g})$ & Summer & $0.8 \pm 0.4$ & $1.0 \pm 0.7$ & $0.8 \pm 0.6$ & $1.8 \pm 0.7$ & * & ns & ns \\
\hline & Autumn & $1.1 \pm 0.8$ & $1.2 \pm 1.0$ & $0.8 \pm 0.4$ & $1.3 \pm 0.9$ & & & \\
\hline Glucose (g) & Summer & $10.6^{\mathrm{a}} \pm 2.8$ & $5.3^{b} \pm 1.4$ & $7.5^{a b} \pm 2.3$ & $6.0^{b} \pm 3.5$ & $* * *$ & ns & ns \\
\hline & Autumn & $10.4^{\mathrm{a}} \pm 3.2$ & $5.0^{\mathrm{b}} \pm 2.2$ & $7.1^{\mathrm{ab}} \pm 2.2$ & $5.8^{\mathrm{b}} \pm 1.8$ & & & \\
\hline Sucrose (g) & Summer & $31.6 \pm 8.3$ & $40.1 \pm 17.4$ & $51.6 \pm 13.7$ & $34.1 \pm 17.9$ & $* *$ & * & ns \\
\hline & Autumn & $40.1 \pm 7.2$ & $43.0 \pm 12.4$ & $61.4 \pm 33.2$ & $43.5 \pm 12.2$ & & & \\
\hline Fructose (g) & Summer & $13.3^{\mathrm{a}} \pm 3.4$ & $6.9^{b} \pm 2.7$ & $9.2^{\mathrm{ab}} \pm 2.6$ & $6.5^{\mathrm{b}} \pm 3.2$ & $* * *$ & ns & ns \\
\hline & Autumn & $12.2^{\mathrm{a}} \pm 4.7$ & $6.8^{b} \pm 2.0$ & $8.7^{\mathrm{ab}} \pm 4.1$ & $7.7^{a b} \pm 2.6$ & & & \\
\hline Lactose (g) & Summer & $2.7^{\mathrm{a}} \pm 2.6$ & $8.1^{b} \pm 3.9$ & $6.2^{\mathrm{ab}} \pm 4.8$ & $7.7^{b} \pm 2.7$ & $* * *$ & ns & ns \\
\hline & Autumn & $4.9^{\mathrm{a}} \pm 3.8$ & $8.6^{\mathrm{ab}} \pm 2.5$ & $6.9^{\mathrm{ab}} \pm 4.1$ & $10.3^{b} \pm 3.7$ & & & \\
\hline Starch $(g)$ & Summer & $62.0^{\mathrm{a}} \pm 12.6$ & $89.1^{\mathrm{ab}} \pm 45.5$ & $82.7^{a} \pm 21.4$ & $124.6^{b} \pm 24.1$ & $* * *$ & ns & * \\
\hline & Autumn & $76.3 \pm 27.3$ & $76.0 \pm 18.3$ & $106.2 \pm 34.8$ & $100.6 \pm 14.9$ & & & \\
\hline Fibre (g) & Summer & $14.7 \pm 2.3$ & $13.2 \pm 2.5$ & $16.1 \pm 4.7$ & $14.5 \pm 3.1$ & * & ns & ns \\
\hline & Autumn & $17.6 \pm 2.7$ & $12.8 \pm 2.1$ & $16.3 \pm 2.6$ & $16.7 \pm 6.4$ & & & \\
\hline Cholesterol (mg) & Summer & $142.6 \pm 73.0$ & $165.4 \pm 125.6$ & $120.4 \pm 88.2$ & $184.2 \pm 92.6$ & ns & ns & ns \\
\hline & Autumn & $187.2 \pm 169.9$ & $163.9 \pm 101.4$ & $108.3 \pm 78.1$ & $183.6 \pm 67.5$ & & & \\
\hline SFA (g) & Summer & $15.2^{\mathrm{ab}} \pm 8.8$ & $11.6^{\mathrm{ab}} \pm 2.4$ & $10.6^{\mathrm{a}} \pm 2.4$ & $20.4^{b} \pm 10.3$ & $* * *$ & ns & ns \\
\hline & Autumn & $13.9^{\mathrm{ab}} \pm 5.0$ & $13.3^{\mathrm{ab}} \pm 3.3$ & $10.7^{\mathrm{a}} \pm 5.4$ & $21.0^{\mathrm{b}} \pm 7.5$ & & & \\
\hline MUFA (g) & Summer & $9.6^{\mathrm{ab}} \pm 3.8$ & $7.7^{\mathrm{a}} \pm 1.7$ & $7.2^{\mathrm{a}} \pm 2.2$ & $12.7^{\mathrm{b}} \pm 4.6$ & $* * *$ & ns & ns \\
\hline & Autumn & $10.7^{\mathrm{ab}} \pm 3.4$ & $9.7^{\mathrm{ab}} \pm 3.2$ & $6.8^{a} \pm 2.7$ & $13.2^{b} \pm 4.8$ & & & \\
\hline
\end{tabular}


Table 1. Cont.

\begin{tabular}{|c|c|c|c|c|c|c|c|c|}
\hline \multirow{2}{*}{$\begin{array}{c}\text { Energy } \\
\text { and Nutrients }\end{array}$} & \multirow{2}{*}{ Season (S) } & \multicolumn{4}{|c|}{ Kindergarten (K) } & \multicolumn{3}{|c|}{ Significant Effects } \\
\hline & & A & B & $\mathrm{C}$ & $\mathbf{D}$ & $\mathbf{K}$ & $S$ & $\mathbf{K} \times \mathbf{S}$ \\
\hline \multirow[t]{2}{*}{ PUFA (g) } & Summer & $2.2^{\mathrm{a}} \pm 0.4$ & $3.2^{\mathrm{ab}} \pm 0.5$ & $3.6^{\mathrm{ab}} \pm 3.0$ & $4.4^{b} \pm 0.9$ & $* * *$ & ns & ns \\
\hline & Autumn & $2.8 \pm 0.6$ & $4.1 \pm 1.5$ & $3.5 \pm 1.6$ & $4.6 \pm 1.1$ & & & \\
\hline \multirow[t]{2}{*}{ PUFA/SFA } & Summer & $0.2 \pm 0.1$ & $0.3 \pm 0.1$ & $0.4 \pm 0.6$ & $0.3 \pm 0.2$ & $*$ & ns & ns \\
\hline & Autumn & $0.2 \pm 0.1$ & $0.3 \pm 0.2$ & $0.4 \pm 0.4$ & $0.2 \pm 0.1$ & & & \\
\hline \multirow[t]{2}{*}{ PUFA n-3 (g) } & Summer & $0.4 \pm 0.1$ & $0.4 \pm 0.1$ & $0.6 \pm 0.5$ & $0.6 \pm 0.2$ & ns & ns & ns \\
\hline & Autumn & $0.5 \pm 0.1$ & $0.5 \pm 0.4$ & $0.5 \pm 0.3$ & $0.7 \pm 0.3$ & & & \\
\hline \multirow[t]{2}{*}{ PUFA n-6 (g) } & Summer & $1.8^{\mathrm{a}} \pm 0.4$ & $2.8^{\mathrm{ab}} \pm 0.4$ & $3.0^{\mathrm{ab}} \pm 2.6$ & $3.8^{\mathrm{b}} \pm 0.8$ & $* * *$ & ns & ns \\
\hline & Autumn & $2.3 \pm 0.6$ & $3.6 \pm 1.4$ & $2.9 \pm 1.5$ & $3.8 \pm 1.0$ & & & \\
\hline \multirow[t]{2}{*}{ PUFA n-6/n-3 } & Summer & $4.4 \pm 1.0$ & $7.4 \pm 1.1$ & $4.6 \pm 1.4$ & $7.1 \pm 2.7$ & $* * *$ & ns & ns \\
\hline & Autumn & $4.7^{\mathrm{a}} \pm 1.8$ & $8.6^{b} \pm 4.3$ & $6.1^{\mathrm{ab}} \pm 2.8$ & $5.7^{\mathrm{ab}} \pm 2.2$ & & & \\
\hline \multirow[t]{2}{*}{ Salt (g) } & Summer & $4.4 \pm 0.8$ & $4.4 \pm 1.0$ & $5.3 \pm 1.1$ & $5.7 \pm 1.9$ & $* *$ & ns & ns \\
\hline & Autumn & $5.4^{\mathrm{ab}} \pm 1.0$ & $4.4^{\mathrm{a}} \pm 0.7$ & $4.9^{\mathrm{ab}} \pm 0.9$ & $6.1^{\mathrm{b}} \pm 1.4$ & & & \\
\hline
\end{tabular}

Significant effects: ${ }^{* *} p<0.001 ;{ }^{* *} 0.001 \leq p<0.01 ; * 0.01 \leq p<0.05$; ns-not significant; K-kindergarten $\mathrm{S}-$ season. $\mathrm{a}-\mathrm{b}$ : Means. Different letters in the same row indicate differences at $\mathrm{p}<0.05$ in view of the kindergarten. SFA—saturated fatty acids, MUFA—-monounsaturated fatty acids, PUFA—polyunsaturated fatty acids.

Table 2. Energy and nutrients in preschool food rations constituting $75 \%$ of the Polish norms and recommendations and the UL established by the EFSA.

\begin{tabular}{|c|c|c|c|c|c|c|c|}
\hline \multirow{2}{*}{$\begin{array}{c}\text { Energy } \\
\text { and Nutrients }\end{array}$} & \multirow{2}{*}{ Norm } & \multirow{2}{*}{$\begin{array}{c}75 \% \\
\text { of the Daily } \\
\text { Requirement }\end{array}$} & \multirow{2}{*}{ Season } & \multicolumn{4}{|c|}{ Norm Realization (\%) } \\
\hline & & & & Kindergarten A & B & $\mathrm{C}$ & D \\
\hline \multirow[t]{2}{*}{ Energy (kcal) } & \multirow[b]{2}{*}{$1400^{1}$} & \multirow[b]{2}{*}{$1050^{1}$} & Summer & $90.0^{\mathrm{a}}$ & $97.6^{\mathrm{a}}$ & $100.9^{\mathrm{a}}$ & $133.1^{\mathrm{b}}$ \\
\hline & & & Autumn & $106.8^{\mathrm{a}}$ & $98.0^{\mathrm{a}}$ & $116.0^{\mathrm{a}}$ & $133.1^{b}$ \\
\hline \multirow[t]{2}{*}{ Protein $(\mathrm{g})$} & \multirow[t]{2}{*}{$21^{2}$} & \multirow[t]{2}{*}{$15.8^{2}$} & Summer & $195.0^{\mathrm{a}}$ & $227.4^{\mathrm{a}}$ & $229.7^{\mathrm{a}}$ & $332.3^{b}$ \\
\hline & & & Autumn & $257.2^{\mathrm{a}}$ & $238.2^{\mathrm{a}}$ & $248.1^{\mathrm{a}}$ & $351.9^{b}$ \\
\hline \multirow[t]{2}{*}{ Fat $(\mathrm{g})$} & \multirow[t]{2}{*}{$47^{3}$} & \multirow[t]{2}{*}{$35.3^{3}$} & Summer & $83.4^{\mathrm{ab}}$ & $69.7^{\mathrm{a}}$ & $66.9^{a}$ & $115.4^{\mathrm{b}}$ \\
\hline & & & Autumn & $85.3^{\mathrm{ab}}$ & $84.1^{\mathrm{ab}}$ & $64.6^{\mathrm{a}}$ & $121.5^{b}$ \\
\hline \multirow[t]{2}{*}{ Carbohydrates (g) } & \multirow[t]{2}{*}{$227.5^{3}$} & \multirow[t]{2}{*}{$170.6^{3}$} & Summer & $86.0^{\mathrm{a}}$ & $100.5^{\mathrm{ab}}$ & $107.7^{\mathrm{ab}}$ & $124.6^{\mathrm{b}}$ \\
\hline & & & Autumn & $106.1^{\mathrm{ab}}$ & $93.4^{\mathrm{a}}$ & $130.2^{b}$ & $120.6^{\mathrm{ab}}$ \\
\hline \multirow[t]{2}{*}{ Sodium (mg) } & \multirow{2}{*}{$1000^{4}$} & \multirow[t]{2}{*}{$750^{4}$} & Summer & 235.1 & 233.0 & 280.1 & 301.5 \\
\hline & & & Autumn & $289.7^{a b}$ & $234.2^{\mathrm{a}}$ & $263.2^{a b}$ & $326.4^{b}$ \\
\hline \multirow[t]{2}{*}{ Potassium (mg) } & \multirow[t]{2}{*}{$1100^{4}$} & \multirow[t]{2}{*}{$825^{4}$} & Summer & 233.7 & 200.2 & 252.8 & 251.0 \\
\hline & & & Autumn & $283.4^{\mathrm{a}}$ & $191.2^{b}$ & $241.0^{\mathrm{ab}}$ & $280 .^{\mathrm{ab}}$ \\
\hline \multirow{2}{*}{ Calcium (mg) } & \multirow[t]{2}{*}{$1000^{2}$} & $750^{2}$ & Summer & $33.4^{\mathrm{a}}$ & $53.6^{\mathrm{ab}}$ & $49.8^{a b}$ & $63.1^{\mathrm{b}}$ \\
\hline & & & Autumn & $48.5^{\mathrm{a}}$ & $50.7^{\mathrm{a}}$ & $48.8^{\mathrm{a}}$ & $83.0^{\mathrm{b}}$ \\
\hline Phosphorus (mg) & $500^{2}$ & $375^{2}$ & Summer & $127.1^{a}$ & $174.1^{\mathrm{ab}}$ & $174.1^{\mathrm{ab}}$ & $219.0^{b}$ \\
\hline & & & Autumn & $171.7^{\mathrm{a}}$ & $181.2^{\mathrm{a}}$ & $180.1^{a}$ & $272.7^{b}$ \\
\hline Magnesium (mg) & $130^{2} / 250^{5}$ & $97.5^{2} / 187.5^{5}$ & Summer & $145.6 / 75.7$ & $174.8 / 90.9$ & $186.9 / 97.2$ & 201.8/105.0 \\
\hline & & & Autumn & 199.9/104.0 & $193.7 / 100.8$ & $184.1 / 95.7$ & $266.1 / 138.4$ \\
\hline Iron (mg) & $10.0^{2}$ & $7.5^{2}$ & Summer & 70.0 & 78.9 & 82.2 & 97.4 \\
\hline & & & Autumn & $93.5^{a b}$ & $82.7^{a}$ & $86.8^{a}$ & $130.2^{b}$ \\
\hline Zinc (mg) & $5.0^{2} / 10.0^{5}$ & $3.8^{2} / 7.5^{5}$ & Summer & $104.8 / 52.4$ & $124.8 / 68.3$ & $124.8 / 62.4$ & $161.5 / 80.8$ \\
\hline & & & Autumn & $144.0 / 72.0$ & $136.6 / 71.3$ & $133.0 / 66.5$ & $180.5 / 90.3$ \\
\hline Copper (mg) & $0.4^{2} / 2.0^{5}$ & $0.3^{2} / 1.5^{5}$ & Summer & $228.9 / 46.0$ & $281.8 / 56.6$ & $274.8 / 54.7$ & $291.5 / 58.0$ \\
\hline & & & Autumn & $285.7 / 57.3$ & $283.5 / 56.7$ & $268.0 / 53.3$ & $331.2 / 66.0$ \\
\hline Manganese (mg) & $1.5^{4}$ & $1.1^{4}$ & Summer & 203.3 & 242.5 & 223.4 & 245.6 \\
\hline & & & Autumn & 244.2 & 265.4 & 223.6 & 305.3 \\
\hline Vitamin $B_{1}(\mathrm{mg})$ & $0.6^{2}$ & $0.5^{2}$ & Summer & 130.7 & 122.1 & 119.0 & 173.6 \\
\hline & & & Autumn & 169.1 & 122.1 & 145.0 & 158.7 \\
\hline Vitamin $B_{2}(\mathrm{mg})$ & $0.6^{2}$ & $0.5^{2}$ & Summer & $143.0^{\mathrm{a}}$ & $203.5^{\mathrm{ab}}$ & $179.3^{\mathrm{ab}}$ & $225.0^{b}$ \\
\hline & & & Autumn & $195.8^{a}$ & $200.3^{a b}$ & $184.8^{a}$ & $267.3^{b}$ \\
\hline Niacin (mg) & $8.0^{2}$ & $6.0^{2}$ & Summer & 130.7 & 89.9 & 136.6 & 173.4 \\
\hline & & & Autumn & $162.3^{\mathrm{ab}}$ & $90.5^{\mathrm{a}}$ & $140.1^{\mathrm{ab}}$ & $199.1^{b}$ \\
\hline Vitamin C (mg) & $50.0^{2}$ & $37.5^{2}$ & Summer & 206.7 & 138.5 & 178.7 & 128.4 \\
\hline & & & Autumn & $261.5^{\mathrm{a}}$ & $99.7^{b}$ & $165.2^{\mathrm{ab}}$ & $146.7^{\mathrm{ab}}$ \\
\hline Vitamin A $(\mu \mathrm{g})$ & $450^{2} / 1100^{5}$ & $337.5^{2} / 825^{5}$ & Summer & $254.0 / 103.9$ & $204.3 / 83.6$ & $224.9 / 92.0$ & $245.3 / 100.4$ \\
\hline & & & Autumn & $289.0^{\mathrm{ab}} / 118.2$ & $187.0^{\mathrm{a} / 76.5}$ & $237.5^{\mathrm{ab}} / 97.2$ & $358.6^{\mathrm{b}} / 146.7$ \\
\hline Vitamin E (mg) & $6.0^{4}$ & $4.5^{4}$ & Summer & 88.0 & 83.1 & 75.0 & 104.9 \\
\hline & & & Autumn & $87.7^{\mathrm{a}}$ & $94.4^{\mathrm{ab}}$ & $82.1^{\mathrm{a}}$ & $123.0^{\mathrm{b}}$ \\
\hline
\end{tabular}


Table 2. Cont.

\begin{tabular}{|c|c|c|c|c|c|c|c|}
\hline \multirow{2}{*}{$\begin{array}{c}\text { Energy } \\
\text { and Nutrients }\end{array}$} & \multirow{2}{*}{ Norm } & \multirow{2}{*}{$\begin{array}{c}75 \% \\
\text { of the Daily } \\
\text { Requirement }\end{array}$} & \multirow{2}{*}{ Season } & \multicolumn{4}{|c|}{ Norm Realization (\%) } \\
\hline & & & & Kindergarten A & B & $\mathrm{C}$ & D \\
\hline \multirow[t]{2}{*}{ Vitamin $\mathrm{B}_{6}(\mathrm{mg})$} & $0.6^{2} / 7.0^{5}$ & $0.5^{2} / 5.3^{5}$ & Summer & $223.3 / 19.1$ & $180.9 / 15.4$ & $238.2 / 20.4$ & $263.3 / 22.5$ \\
\hline & & & Autumn & $273.6^{\mathrm{a}} / 23.4$ & $169.7^{\mathrm{b}} / 14.5$ & $223.1^{\mathrm{ab}} / 19.1$ & $275.0^{\mathrm{a}} / 23.6$ \\
\hline \multirow[t]{2}{*}{ Vitamin D ( $\mu g)$} & $15.0^{4}$ & $11.3^{4}$ & Summer & 6.9 & 8.5 & 7.2 & 15.6 \\
\hline & & & Autumn & 9.9 & 10.5 & 7.2 & 11.7 \\
\hline \multirow[t]{2}{*}{ Glucose (g) } & & & Summer & $40.2^{\mathrm{a}}$ & $20.3^{b}$ & $28.7^{\mathrm{ab}}$ & $23.0^{b}$ \\
\hline & & & Autumn & $39.8^{a}$ & $19.2^{b}$ & $26.9^{a b}$ & $21.9^{b}$ \\
\hline \multirow[t]{2}{*}{ Sucrose (g) } & * $10 \%$ energy & 26.3 & Summer & 120.2 & 152.8 & 196.7 & 130.0 \\
\hline & value & & Autumn & 152.8 & 163.7 & 234.0 & 165.8 \\
\hline \multirow[t]{2}{*}{ Fructose (g) } & & & Summer & $50.6^{a}$ & $26.3^{b}$ & $35.2^{a b}$ & $24.7^{\mathrm{b}}$ \\
\hline & & & Autumn & $46.3^{a}$ & $25.9^{b}$ & $33.1^{\mathrm{ab}}$ & $29.1^{\mathrm{ab}}$ \\
\hline \multirow[t]{2}{*}{ Fiber $(\mathrm{g})$} & $14^{4}$ & $10.5^{4}$ & Summer & 140.4 & 125.3 & 153.2 & 137.6 \\
\hline & & & Autumn & 167.6 & 122.1 & 154.8 & 158.6 \\
\hline \multirow[t]{2}{*}{ Cholesterol (mg) } & 300 & 225 & Summer & 63.4 & 73.5 & 53.5 & 81.9 \\
\hline & & & Autumn & 83.2 & 72.9 & 48.1 & 81.6 \\
\hline \multirow[t]{2}{*}{ SFA $(g)$} & 15.6 & 11.7 & Summer & $130.1^{\mathrm{ab}}$ & $98.9^{a b}$ & $90.5^{\mathrm{a}}$ & $174.7^{\mathrm{b}}$ \\
\hline & & & Autumn & $119.2^{a b}$ & $113.8^{a b}$ & $91.3^{\mathrm{a}}$ & $179.8^{b}$ \\
\hline \multirow[t]{2}{*}{ PUFA n-3 (g) } & $1.0^{4}$ & $0.8^{4}$ & Summer & 54.8 & 49.4 & 82.4 & 76.3 \\
\hline & & & Autumn & 63.5 & 68.4 & 64.6 & 95.5 \\
\hline \multirow[t]{2}{*}{ PUFA n-6 (g) } & $6.2^{4}$ & $4.7^{4}$ & Summer & $39.0^{a}$ & $60.1^{\mathrm{ab}}$ & $64.2^{\mathrm{ab}}$ & $81.2^{b}$ \\
\hline & & & Autumn & 49.0 & 77.1 & 61.0 & 81.8 \\
\hline \multirow[t]{2}{*}{ Salt (g) } & 2.5 & 1.9 & Summer & 234.7 & 232.5 & 279.3 & 301.1 \\
\hline & & & Autumn & $289.1^{\mathrm{ab}}$ & $233.5^{\mathrm{a}}$ & $262.8^{a b}$ & $325.0^{b}$ \\
\hline
\end{tabular}

* $10 \%$ energy value of food ratio; ${ }^{1}$ Estimated Energy Requirement (EER); ${ }^{2}$ Recommended Dietary Allowance (RDA); ${ }^{3}$ Reference Intake ranges (RIs); ${ }^{4}$ Adequate Intake (AI); ${ }^{5}$ Tolerable Upper Intake Level (UL). SFA — saturated fatty acids, MUFA - monounsaturated fatty acids, PUFA - polyunsaturated fatty acids. a-b: Means. Different letters in the same row indicate differences at $p<0.05$ in view of the kindergarten.

The protein content in the analyzed preschool rations ranged from $30.7 \mathrm{~g}$ to $52.3 \mathrm{~g}$ in summer and from $37.5 \mathrm{~g}$ to $55.4 \mathrm{~g}$ in autumn, respectively (Table 1 ). This amount of protein exceeded the recommended level of consumption (intake above $75 \%$ of RDA) by two or even three times (Table 2). The menus from kindergarten D had a significantly higher content of this nutrient compared to the other kindergartens (Table 2).

The food ration of the children who spend part of the day in kindergarten should provide $35.3 \mathrm{~g}$ of fat. In this study, this amount was exceeded by $15.4 \%$ in summer and $21.5 \%$ in autumn in kindergarten D (Table 2). In the remaining kindergartens, the fat content was lower than recommended. From the nutritional point of view, it is also important to identify the type of fatty acids in the diet. In each kindergarten, the children's diet was characterized by too low n-3 and n- 6 polyunsaturated fatty acid content. An excess of unsatisfactory saturated fatty acids was found in kindergartens A and D in both seasons (Table 2).

The carbohydrate content differed significantly between preschools. The menus were balanced in terms of carbohydrate content in kindergartens B and C in summer and A and $B$ in autumn (Table 2). An excessive supply of carbohydrates in summer and autumn was recorded in kindergarten $\mathrm{D}$ (Table 2). In the assessment of the supply of added sugars (sucrose, fructose, glucose), it was assumed that they should constitute no more than $10 \%$ of the diet's energy. Regardless of the kindergarten, the sucrose content exceeded the recommended intake (Table 2). The proportion of carbohydrates from each group in the children's meals was inappropriate (Table 3). The children's diet was characterized by a too low proportion of starch-containing products. The dietary fiber content was above the recommended level in each facility (Table 2).

The analyzed menus, compared to the norms, covered the children's needs for minerals (such as sodium, potassium, calcium, phosphorus, magnesium, calcium, iron, zinc, copper, manganese) to varying degrees. Regardless of the kindergarten, a significant calcium inadequacy was noted in the studied menus (Table 2). Its content in summer ranged from $250.4 \mathrm{mg}$ to $473.2 \mathrm{mg}$, and in autumn, from $364.0 \mathrm{mg}$ to $622.3 \mathrm{mg}$ (with the recommended standard of $750 \mathrm{mg}$ ) (Table 1). 
Table 3. Percentage of carbohydrate groups in total carbohydrate content in kindergarten menus.

\begin{tabular}{cccccc}
\hline \multirow{2}{*}{$\begin{array}{c}\text { Type of } \\
\text { Carbohydrates }\end{array}$} & Season & A & B indergatren \\
\cline { 3 - 6 } & & 7.2 & 3.1 & C & D \\
\hline \multirow{2}{*}{ Glucose } & Summer & 5.8 & 2.9 & 4.1 & 2.8 \\
& Autumn & 21.5 & 23.4 & 28.2 & 2.8 \\
Sucrose & Summer & 22.2 & 27.0 & 27.6 & 16.1 \\
& Autumn & 9.1 & 4.0 & 5.0 & 21.1 \\
Fructose & Summer & 6.7 & 4.3 & 3.9 & 3.1 \\
& Autumn & 1.8 & 4.8 & 3.4 & 3.7 \\
Lactose & Summer & 2.7 & 5.4 & 3.1 & 3.6 \\
& Autumn & 42.3 & 52.0 & 45.0 & 5.0 \\
& Summer & 42.2 & 47.7 & 47.8 & 58.6 \\
\end{tabular}

The iron content in kindergartens $B$ and $C$ summer and autumn menus was also below $75 \%$ of the RDA. The daily supply of this mineral exceeded $75 \%$ of RDA in kindergarten $\mathrm{D}$ in autumn (Table 2). At the same time, it should be emphasized that UL standards for iron have not been determined [35]. The content of the remaining analyzed minerals (sodium, potassium, phosphorus, magnesium, zinc, copper, and manganese) in all the analyzed menus was too high according to Polish standards (RDA, AI) (Table 2). It should also be noted that the intake of magnesium was higher than $75 \%$ of the UL in the fall in the kindergartens $\mathrm{A}$ and $\mathrm{B}$ and in institution $\mathrm{D}$ in both seasons, and for vitamin $\mathrm{A}$ in kindergartens $A$ and $D$. The UL levels for zinc, copper, and vitamin $B_{6}$ were not exceeded.

In the analyzed preschool menus there were irregularities in the vitamin content. $\mathrm{A}$ vitamin D deficiency (intake lower than $75 \%$ of $\mathrm{AI}$ ) and an excess of vitamins $\mathrm{A}, \mathrm{B}_{1}, \mathrm{~B}_{2}$, and $\mathrm{B}_{6}$ (intakes above $75 \%$ of RDA) were demonstrated in each preschool (Table 2). The supply of niacin and vitamins $C$ and $E$ varied depending on the kindergarten (Table 2).

In all menus, there were irregularities in the salt content. The content in preschool rations ranged from $232.5 \mathrm{~g}$ to $325.0 \mathrm{~g}$, respectively (Table 1 ). This amount of salt was twice or even three times higher than the recommended intake level (Table 2).

The all-day food ration in the kindergarten consisted of 3 meals: breakfast, lunch, and afternoon tea. The intervals between meals were adequate at 4 and $3 \mathrm{~h}$, respectively.

Regardless of the kindergarten, breakfast was usually served with wheat bread, butter, and a high-protein product of animal origin (cold cuts of meat, eggs, cheese) or an item with high carbohydrate content such as jam (most often strawberry). Children were also served milk with corn flakes or other cereals. To drink, tea, grain coffee, or cocoa were provided. The drinks were sweetened with sugar. Each breakfast was served with a piece of fruit (most often apples, bananas) or a vegetable (most often cucumbers or tomatoes).

Lunch always consisted of two dishes: soup and a second course. The most frequently served soups included tomato, potato, barley, or chicken, and cabbage. They were prepared based on meat and vegetable stock. They were not enriched with fresh dill or parsley. The second course usually consisted of a protein product of animal origin-usually meat, a bulk product-mainly potatoes and less often pasta or rice, as well as cooked or raw vegetables. Compote with sugar or fruit and vegetable drinks were usually served with lunch.

Afternoon tea usually consisted of products rich in carbohydrates (buns, jelly, bread with chocolate spread, honey) and a warm drink (tea, milk, or milk drinks with added sugar). Sometimes sandwiches were served (with pate, pork sirloin, sausage, or jam).

The meals were prepared in a variety of techniques. The products were fried, boiled, stewed, or baked. The meals were composed with contrasts in taste, smell, and color to encourage children to eat. Combining two dishes of a similar nature (sweet, sour, or similar in color and texture) was avoided during one meal. No seasonality of products was found in the menus. The disadvantage of the analyzed menus was the lack of whole grain products, the limited quantities of fish and legumes, and the servings of meat products with high fat content (mortadella, sausages, and cheese). Spices were added in limited amounts. 


\section{Discussion}

The statistical analysis of the obtained results showed that the energy value of the studied menus differed regarding the season and the kindergarten. In autumn, a favorable tendency of an energy increase in menus was observed when the body's energy expenditure related to the need to maintain the body temperature at about $37^{\circ} \mathrm{C}$ increased, with a simultaneous decrease in the surrounding temperature. The energy values of the kindergarten meals, except for facility D and C (in autumn), were correct. Preschool meals should cover the energy and nutrient needs of children to a greater extent than home meals. However, they should not exceed the recommendations because they lead to, among other issues, excess weight and obesity. The results of this study do not differ from the data in the literature on the nutrition of children in kindergartens [20,23,27,37-39], which report too high energy supply as compared to the recommendations.

Kindergarten D also reported excess fat in the children's diet, which probably came from chocolate and confectionery products, which provided 10 to $15 \mathrm{~g}$ of fat per $100 \mathrm{~g}$ of product [32] and were also a source of unfavorable saturated fatty acids. The consumption of products rich in fat, including saturated fatty acids, is associated with the risk of ischemic heart disease and the development of cancer, mainly colorectal and breast cancer [40-42].

The average protein content of the evaluated food rations from the analyzed kindergartens was more than double the RDA norm. Similar results in preschool diets were also obtained by the other authors $[22,27,30,43]$. Such a protein supply was determined by the high consumption of meat and processed meat products. So far, an UL standard has not been defined for protein but attempts are underway to determine the upper limit of consumption. However, it is known that high protein consumption may be associated with hypercalciuria, resulting in osteoporosis, acidosis, and an increased risk of kidney stones $[4,44]$. Excess dietary protein content in childhood contributes to an increased risk of obesity [45].

An analysis of the data in the literature shows that there is a lack of studies on the intake of specific groups of carbohydrates, including fructose, which is extremely important because of its negative impact on human health [46-48]. The increased consumption of products (sweetened soft drinks, candies) to which fructose is added (especially as a glucose-fructose syrup [4]) during the production process was observed. The highest fructose content was found in the meals of kindergarten A. Excessive fructose intake is believed to be one of the factors responsible for the steady increase in excess weight and obesity problems in modern societies [49]. This is especially true in children and adolescents [50].

One of the tools to support the improvement of the nutrition of children and adolescents in educational institutions was the Regulation of the Minister of Health of 26 July 2016 on the groups of food intended for sale to children and adolescents within the educational system and the requirements for food served as part of the collective nutrition of children and adolescents in educational settings (Journal of Laws of 2016, item 1154) [51]. The provisions of the regulation covered, among others, requirements for collective nutrition in kindergartens, school establishments, boarding schools, and boarding houses. The regulation requires the inclusion of more vegetables and fruit so that they are present every day in each meal, with the inclusion of more fish in the weekly diet while limiting the addition of sugar in drinks prepared onsite to a maximum of $10 \mathrm{~g}$ per $250 \mathrm{~mL}$ of drink. This was designed to enable easier compliance with the standards' recommendation to limit sugars (sucrose, fructose, glucose) added to food during its manufacture and production, which should provide no more than $10 \%$ of energy.

Despite the above-mentioned regulation, in each kindergarten the amount of sugar added to food during its production process exceeded recommendations. Sucrose content ranged from $31.6 \mathrm{~g}$ to $61.4 \mathrm{~g}$, corresponding to $120 \%$ and $234.0 \%$ of the standard value, which is a disturbing phenomenon. It is well known that an excessive intake of sucrose and simple sugars contributes to the occurrence of many chronic non-communicable diseases, including excess weight and obesity problems, insulin resistance, diabetes, metabolic 
syndrome, tooth decay, cancer, and non-alcoholic fatty liver disease [52-55]. Other authors have also noted high sucrose content in the diet of preschool children [24,27].

It should be noted that from 1 January 2021, regulations entered into force in Poland introducing taxes on sweetened and energy drinks (ACT of 14 February 2020 on the amendment of certain acts in connection with the promotion of pro-health consumer choices) [56]. Perhaps this regulation will reduce the sale and consumption of sweetened beverages and improve the health indicators of the Polish society.

The mineral content of the study rations varied widely. In all the evaluated dietary rations from the studied kindergartens, the calcium content was too low compared to the standard. The low supply of calcium in the analyzed dietary rations was caused by the low supply of milk and milk products (e.g., cottage cheese, kefir, buttermilk), which represent the main sources of calcium in the diet. Adequate calcium content is particularly desirable in the diet of small children because calcium is an important building material for bones and teeth [57,58]; it is also involved in muscle contraction, blood coagulation, conduction of nerve impulses, activation of enzymes, and the reduction in the permeability of cell membranes, as well as supporting the proper functioning of the cardiovascular system. Calcium inadequacy in children's diets is a common problem [28-30,59-61]. The utilization of calcium from the diet is hindered by a too-high phosphorus content [62]. An adequate calcium/phosphorus ratio is a very significant factor in calcium phosphate metabolism. A too low calcium/phosphorus ratio (indicating a high dietary phosphorus intake) stimulates parathormone secretion and enhances calcium resorption from the bones. The calcium-to-phosphorus ratio was abnormal in the children's diets, ranging between 0.5 to 0.6 regardless of the preschool institution and a season.

It should also be mentioned that the factors increasing calcium absorption include, among others, vitamin D, the amount of which in the analyzed diets was significantly below the norm. Insufficient intake of calcium and vitamin D raises serious concerns about the proper condition of children's skeletons. The results of this research indicate that it is necessary to educate the employees of preschool institutions responsible for children's nutrition. Social campaigns promoting the importance of calcium intake and indicating food products and their quantities needed to meet the child's demand for this mineral may be a way to draw public attention to the consequences of calcium deficiency in children.

Based on the research results, it was found that, regardless of the kindergarten, the sodium content in the children's diet was very high (intake higher than $75 \%$ of AI). Such high sodium intake indicates the need to limit the addition of salt to dishes and the elimination of processed products with a high salt content (e.g., cold meat products). The results of sodium consumption converted into table salt exceeded the recommendations by more than 2-3 times. There is no doubt that salt consumption in Poland should be reduced $[2,3,6]$. It is advisable to develop proper eating habits from an early age to reduce the risk of developing cardiovascular diseases, arterial hypertension, and cancer of the stomach and throat at a later age $[63,64]$. Many studies report excessive sodium and table salt intake in children's diets, suggesting that it is a common phenomenon [30,59,65-67].

Regardless of the kindergarten, the analyzed food rations also contained too high intakes of potassium, copper, manganese, and magnesium compared to the Polish norms. It should be noted that EFSA experts concluded that there is currently insufficient scientific research on the negative effects of the excess of sodium, potassium, and manganese on the human body [35]. Consequently, it is currently impossible to establish ULs for these components. The UL for copper intake was not exceeded. In this situation, the intake of potassium, copper, and manganese is satisfactory. The intake of magnesium was higher than $75 \%$ of UL. Doses of magnesium above $250 \mathrm{mg}$ /day have laxative properties [4].

The analyzed food rations covered the children's iron needs to varying degrees. In kindergartens $B$ and $C$, iron deficiency was demonstrated, while in kindergarten D an excess was found. At the same time, it should be emphasized that UL standards for iron have not been determined [35]. It is well known that iron deficiency promotes the development of anemia [68] and may reduce a child's immunity [69]. At the same time, its excess 
increases the risk of cancer, gastric disorders, atherosclerosis, strokes, and Alzheimer's and Parkinson's disease [70-72]. Literature data also show large variations in iron consumption among children, usually insufficient $[28,30,39,67,73]$ or exceeding the norm $[22,43]$.

Regardless of the kindergarten, the children's food rations showed an excess of vitamins $A, B_{1}, B_{2}, B_{6}$, and $C$ (except for kindergarten $B$ ) and vitamin $D$ deficiency. The study results do not differ from the results of the studies by the other authors who found excessive intake of vitamin A in preschool food rations compared to the norms [28,30,43]. An excessive supply of vitamin A manifests itself in irritability, headaches, loss of appetite, dry skin, and digestive tract disorders, which are accompanied by enlargement of the liver and spleen [1]. Since the UL values have not been established for vitamins $B_{1}, B_{2}$ and $C$ and the UL value standard for vitamin $B_{6}$ was not exceeded, their intake level can be assessed positively.

Children's food rations were characterized by a deficient supply of vitamin $\mathrm{D}$, whose deficiency may result in the negative calcium balance and impaired bone mineralization, resulting in rickets in children [74]. Deficiency of this vitamin was due to a very low intake of fish, which was served sporadically, regardless of the preschool. Vitamin D is found in smaller amounts in butter, yellow cheese, egg yolk, and meat, which were served to the children in meals. However, with the deficient intake of fish, the other listed sources of the vitamin did not cover the children's vitamin $\mathrm{D}$ requirements.

Numerous studies indicate irregularities in the nutrition of children in kindergartens. The most frequent recurring problem is an excessive supply of fat, vitamin A, salt, and calcium, and vitamin D deficiency, which is also confirmed by the results of this study.

It should be mentioned that the data on the energy and nutritional value of children diet were obtained from the tables of composition and nutritional value [32]. The tables' values are averaged data, so the energy and nutrient content of a product consumed by children may differ from the values given in the tables. The content can depend on many factors, both genetic and environmental. The limitation of this study is the lack of data regarding the real food consumption of children, food waste on the plate, and the real amount of salt added to dishes. Consequently, the estimated content of energy and nutrients in the daily food rations of children may differ from their actual consumption. Thus, the observed deficiencies of the selected nutrients in the children's diets are hazardous, as their actual consumption may be even lower. It is essential to be aware that preschool nutrition represents only a part of the diet. It is not known what foods products the children received at home and whether their parents introduced supplements into their diet. It is worth mentioning that the menu data were acquired through 2019 only. However, they are still valid, as during 2020 and 2021 there were lockdowns, and kindergartens were closed. Therefore, there were more important issues to combat than kindergarten menu adjustment to the proper nutrition schemes.

\section{Conclusions}

The adequate nutrition of children is necessary to ensure their proper development. The evaluation of preschool food rations is important to prevent nutrition errors in this group. The study results indicate that the analyzed preschools did not fully implement the requirements set out in the Regulation of the Ministry of Health of 26 July 2016 [51]. Not every kindergarten meal included vegetable or fruit. More than two portions of fried food were served per week. The children's diet did not contain nuts. Fish was served sporadically. One of the disadvantages of the analyzed menus was the presence of sugary foods. Chocolate, honey, and cornflakes with added sugar were often served for breakfast. The afternoon snack was served with confectionery products such as buttered buns and puff pastries. As is commonly known, sweets in a child's diet should be limited to a minimum, and such an institution as a kindergarten should provide children with proper models concerning nutrition. No seasonality of products was noticed in the menus. The same fruit and vegetables were served in summer and autumn. The assessment of menus, regardless of the kindergarten, showed that they were arranged incorrectly, which indicates the need 
for modification of their composition. Therefore, it is necessary to carry out nutritional education among kindergarten staff. To ensure the health of Polish children and thus the health of future society, it is necessary to correct dietary mistakes as soon as possible, since diet-related diseases may develop from childhood.

Funding: This research received no external funding.

Institutional Review Board Statement: Not applicable.

Informed Consent Statement: Not applicable.

Data Availability Statement: Not applicable.

Acknowledgments: The author thanks Joanna Harasym for manuscript editing consultation and Dorota Matysiak, an employee of the Main Library of the Wroclaw University of Economics and Business, for help in searching for literature to prepare this work.

Conflicts of Interest: The author declares no conflict of interest.

\section{References}

1. Sadowska, J.; Radziszewska, M.; Krzymuska, A. Evaluation of nutrition manner and nutritional status of pre-school children. Acta Sci. Pol. Technol. Aliment. 2010, 9, 105-115.

2. Myszkowska-Ryciak, J.; Harton, A. Nutrition-related practices in kindergartens in the context of changes to legal regulations on foodstuffs used in canteen menus for children. Rocz. Panstw. Zakl. Hig. 2018, 69, 31-36.

3. Merkiel, S.; Chalcarz, W. Preschool diets in children from Piła, Poland, require urgent intervention as implied by high risk of nutrient inadequacies. J. Health Popul. Nutr. 2016, 35, 11. [CrossRef]

4. Jarosz, M.; Rychlik, E.; Stoś, K.; Charzewska, J. Nutrition Standards for the Population of Poland and Their Application; National Institute of Public Health-National Institute of Hygiene: Warsaw, Poland, 2020.

5. Charzewska, J. Recommendations for Nutrition Implementers in the Field of Proper Nutrition of Children in Kindergartens; Food and Nutrition Institute: Warsaw, Poland, 2011.

6. Merkiel, S.; Chalczarz, W. Review of the studies on nutrition in polish preschool children. Part 1. Preschool menus. Rocz. Panstw. Zakl. Hig. 2016, 67, 223-235. [PubMed]

7. Stroba, M.; Malinowska-Borowska, J. Assessment of calcium and vitamin d content in meals served for preschool children attending to kindergartens in some Silesian cities. Rocz. Panstw. Zakl. Hig. 2021, 72, 83-88.

8. Myszkowska-Ryciak, J.; Harton, A. Evaluation of implementation of dietary recommendations and guidelines in selected kindergartens from Poznan area. Probl. Hig. Epidemiol. 2018, 99, 7-11.

9. Klemarczyk, W.; Strucińska, M.; Weker, H.; Więch, M. Dietary assessment of children in vegetarian kindergarten. Pediatria współczesna. Gastroenterol. Hepatol. I Zyw. Dziecka 2005, 7, 243-246.

10. Dymkowska Malesa, M.; Szparaga, A. Evaluation of intake of selected vitamins and minerals in the diets of preschool children from Koszalin. Nowa Pediatr. 2013, 3, 106-110.

11. Stempel, P.; Galczak-Kondraciuk, A.; Czeczelewski, J.; Kołdej, M. Assessment of nutritional behaviour of children aged 3-7 from selected kindergartens in Biala Podlaska county. Rocz. Panstw. Zakl. Hig. 2018, 69, 289-297. [PubMed]

12. World Health Organization. Prevalence of Obesity among Children and Adolescents, BMI $>+2$ Standard Deviations above the Median (Crude Estimate) (\%). Available online: https://www.who.int/data/gho/data/indicators/indicator-details/GHO/ prevalence-of-obesity-among-children-and-adolescents-bmi-2-standard-deviations-above-the-median-(crude-estimate)-(-) (accessed on 10 December 2021).

13. Lobstein, T.; Jackson-Leach, R. Planning for the worst: Estimates of obesity and comorbidities in school-age children in 2025. Pediatr. Obes. 2016, 11, 321-325. [CrossRef]

14. WHO. Reducing Childhood Obesity in Poland Final WEB.pdf (who.int). Available online: https://www.euro.who.int/__data/ assets/pdf_file/0011/350030/Reducing-childhood-obesity-in-Poland_final_WEB.pdf (accessed on 10 December 2021).

15. Noczyńska, A.; Zubkiewicz-Kucharska, A. The influence of birth weight and parents' weight on overweight and obesity prevalence among their children. Pediatr. Endocrinol. 2014, 13, 23-30. [CrossRef]

16. Stapor, N.; Kapczuk, I.; Krzewska, A.; Sieniawska, J.; Rakuś-Kwiatosz, A.; Piątek, D.; Bąk, K.; Szydełko, J.; Beń-Skowronek, I. What is the difference in lifestyle in slim and overweight children? Pediatr. Endocrinol. 2016, 15, 29-35. [CrossRef]

17. Llewellyn, A.; Simmonds, M.; Owen, C.G.; Woolacott, N. Childhood obesity as a predictor of morbidity in adulthood: A systematic review and meta-analysis. Obes. Rev. 2016, 17, 56-67. [CrossRef] [PubMed]

18. Batko, B.; Kowal, M.; Szwajca, M.; Pilecki, M. Relationship between biopsychosocial factors, body mass and body composition in preschool children. Psychiatr. Psychol. Klin. 2020, 20, 164-173. [CrossRef]

19. Rolland-Cachera, M.F.; Deheeger, M.; Bellisle, F.; Sempé, M.; Guilloud-ataille, M.; Patois, E. Adiposity rebound in children: A simple indicator for predicting obesity. Am. J. Clin. Nutr. 1984, 39, 129-135. [CrossRef] 
20. Grajeta, H.; Ilow, R.; Prescha, A.; Regulska-Ilow, B.; Biernat, J. Evaluation of energy and nutritive value of nursery schools' meals. Rocz. Panstw. Zakl. Hig. 2003, 54, 417-425.

21. Leszczyńska, T.; Sikora, E.; Kręcina, K.; Pysz, K. Meals served in nursery schools and their share in meeting the recommended daily demand for energy and nutrients exemplified by one selected canteen. Żywność Nauka Technol. Jakość 2007, 6, 327-334.

22. Michota-Katulska, E.; Zegan, M. Comperative analysis of children of nutrition in kindergartens in the traditional system and catering. Med. Rodz. 2014, 4, 166-169.

23. Czech, A.; Kęska, A. Nutrient content in preschool rations in spring and autumn. Żyw. Człow. Metab. 2007, 34, 561-571.

24. Rogalska-Niedźwiedź, M.; Charzewska, J.; Chabros, E.; Chwojnowska, Z.; Wajszczyk, Z.; Zachrewicz, E. Nutrition of 4-year-old children from rural and urban environments. Probl. Hig. Epidemiol. 2008, 89, 80-84.

25. Kowieska, A.; Biel, W.; Chalaba, A. Characteristics of the nutrition of preschool children. Żyw. Człow. Metab. 2009, 36, 179-184.

26. Dymkowska-Malesa, M.; Skibniewska, K.A. Meals served at nursery schools and their share in meeting the recommended daily demand for nutrients and energy Bromat. Chem. Toksykol. 2011, 44, 374-379.

27. Frąckiewicz, J.; Ring-Andrzejczuk, K.; Gronowska-Senger, A. Energy and selected nutrients content in pre-school children diet of Warsowia district. Roczn. Panstw. Zakl. Hig. 2011, 62, 181-185.

28. Orkusz, A.; Olech, A. Evaluation of the nutritional value of nursery school meals. Eng. Sci. Technol. 2014, 2, 77-87. [CrossRef]

29. Orkusz, A.; Włodarczyk, A. Assessment of preschool children’s decades menus. Eng. Sci. Technol. 2014, 1, 72-81. [CrossRef]

30. Kwiecień, M.; Winiarska-Mleczan, A.; Danek-Majewska, A.; Kiczorowska, B.; Olcha, M. Assesment of nutritional value of feed rations for preschool children with particular emphasis on mineral content. Probl. Hig. Epidemiol. 2015, 96, 742-745.

31. Wrzochal, A.; Gładyś-Jakubczyk, A.; Edyta Suliga, E. Evaluation of diet in preschool-age children with Down syndromePreliminary examination. Med. Stud. 2019, 35, 128-138. [CrossRef]

32. Computer Program Diet 6D, Independent Laboratory of Epidemiology and Nutrition Standards; Institute of Food and Nutrition: Warsaw, Poland, 2018.

33. Kunachowicz, H.; Nadolna, I.; Przygoda, B.; Iwanow, K. Tables of Composition and Nutritional Value of Food; PZWL: Warsaw, Poland, 2005.

34. Kułaga, Z.; Grajda, A.; Gurzkowska, B.; Góźdź, M.; Wojtyło, M.; Świąder, A.; Różdżyńska-Świątkowska, A.; Litwin, M. Polish 2012 growth references for preschool children. Eur. J. Pediatr. 2013, 172, 753-761. [CrossRef] [PubMed]

35. European Food Safety Authority (EFSA). Summary of Tolerable Upper Intake Levels. September 2018; pp. 1-4. Available online: https://www.efsa.europa.eu/sites/default/files/assets/UL_Summary_tables.pdf (accessed on 10 December 2021).

36. Statistica, Version 13.3; StatSoft Inc.: Kraków, Poland, 2013.

37. Kłos, A.; Bertrand, J. Nutrition of children in selected military kindergartens in Warsaw. Lek. Wojsk. 1999, 5, 275-279.

38. Hamułka, J.; Wawrzyniak, A. Nutritional assessment of decadal menus of children aged 1-6 years. Bromat. Chem. Toksykol. 2003, 36, 7-11.

39. Orkusz, A.; Janczar-Smuga, M.; Frysiak, D. Assessment of nutrition of children aged 4-6 years basing on decade menus. Zesz. Probl. Post. Nauk. Roln. 2018, 594, 37-47. [CrossRef]

40. Enos, R.T.; Velázquez, K.T.; McClellan, J.L.; Cranford, T.L.; Nagarkatti, M.; Nagarkatti, P.S.; Davis, J.M.; Murphy, E.A. High-fat diets rich in saturated fat protect against azoxymethane/dextran sulfate sodium-induced colon cancer. Am. J. Physiol. Gastrointest. Liver Physiol. 2016, 310, G906-G919. [CrossRef] [PubMed]

41. Nettleton, J.A.; Brouwer, I.A.; Geleijnse, J.M.; Hornstra, G. Saturated Fat Consumption and Risk of Coronary Heart Disease and Ischemic Stroke: A Science Update. Ann. Nutr. Metab. 2017, 70, 26-33. [CrossRef] [PubMed]

42. Sieri, S.; Chiodini, P.; Agnoli, C.; Pala, V.; Berrino, F.; Trichopoulou, A.; Benetou, V.; Vasilopoulou, E.; Sánchez, M.-J.; Chirlaque, M.-D.; et al. Dietary Fat Intake and Development of Specific Breast Cancer Subtypes. JNCI 2014, 106, dju068. [CrossRef]

43. Orkusz, A.; Hapanowicz, K. Assessment of the energy and nutritional values of meals in selected kindergarten in Wroclaw. Eng. Sci. Technol. 2016, 4, 85-94. [CrossRef]

44. Delimaris, J. Adverse effects associated with protein intake above the recommended dietary allowance for adults. ISRN Nutr. 2013, 2013, 1-6. [CrossRef] [PubMed]

45. Campbell, K.J.; Abott, G.M.P.H.; Zheng, M.; Mc Naughton, S.A. Early Life Protein Intake: Food Sources, Correlates, and Tracking across the First 5 Years of Life. J. Acad. Nutr. Diet. 2017, 117, 1188-1197. [CrossRef]

46. Gaby, A.R. Adverse effects of dietary fructose. Altern. Med. Rev. 2005, 10, 294-306. [PubMed]

47. Federico, A.; Rosato, V.; Masarone, M.; Torre, P.; Dallio, M.; Romeo, M.; Persico, M. The Role of Fructose in Non-Alcoholic Steatohepatitis: Old Relationship and New Insights. Nutrients 2021, 13, 1314. [CrossRef]

48. Stricker, S.; Rudloff, S.; Geier, A.; Steveling, A.; Roeb, E.; Zimmer, K.P. Fructose consumption-free sugars and their health effects. Dtsch. Arztebl. Int. 2021, 5, 71-78. [CrossRef]

49. Okreglicka, K.; Pardecki, M.; Jagielska, A.; Tyszko, P.Z. Metabolic effects of excessive dietary fructose intake. Med. Og. Nauk. Zdr. 2017, 23, 165-170. [CrossRef]

50. Kretowicz, M.; Goszka, G.; Brymora, A.; Flisiński, M.; Odrowąż-Sypniewska, G.; Manitius, J. Is there a relationship among daily fructose intake, blood pressure and uric acid level in chronic kidney disease patients without diabetes? Arter. Hypertens. 2011, 15, 341-346.

51. Regulation of the Polish Minister of Health of 26 July 2016 on the Groups of Foodstuffs Intended for Sale to Children and Adolescents in Units of the Educational System and the Requirements to Be Met by Foodstuffs Used as a Part of Collective Nutrition of Children and Adolescents in These Units (Dz.U. from 2016 poz. 1154; 01.08.2016). Available online: https://isap.sejm.gov.pl/isap.nsf/ DocDetails.xsp?id=WDU20160001154 (accessed on 10 December 2021). 
52. Sartorius, B.; Sartorius, K.; Aldous, C.; Madiba, T.E.; Stefan, C.; Noakes, T. Carbohydrate intake, obesity, metabolic syndrome and cancer risk? A two-part systematic review and meta-analysis protocol to estimate attributability. BMJ Open 2016,6 , e009301. [CrossRef] [PubMed]

53. Stanhope, K.L. Sugar consumption, metabolic disease and obesity: The state of the controversy. Crit. Rev. Clin. Lab. Sci. 2016, 53, 52-67. [CrossRef] [PubMed]

54. Sheiham, A.; James, W.P. A new understanding of the relationship between sugars, dental caries, and fluoride use: Implications for limits on sugars consumption. Public Health Nutr. 2014, 17, 2176-2184. [CrossRef]

55. Delli Bovi, A.P.; Di Michele, L.; Laino, G.; Vajro, P. Obesity and Obesity Related Diseases, Sugar Consumption and Bad Oral Health: A Fatal Epidemic Mixtures: The Pediatric and Odontologist Point of View. Transl. Med. UniSa. 2017, $16,11-16$.

56. Polish Act of February 14, 2020 on the Amendment of Certain Acts in Connection with the Promotion of Pro-Health Consumer Choices. (Dz.U. from 2020 poz. 1492; 31.08.2020). Available online: https:/ / isap.sejm.gov.pl/isap.nsf/DocDetails.xsp?id=WDU2 0200001492 (accessed on 10 December 2021).

57. Bueno, A.L.; Czepielewski, M.A. The importance for growth of dietary intake of calcium and vitamin D. J. Pediatr. 2008, 84, 386-394. [CrossRef]

58. Dermience, M.; Lognay, G.; Mathieu, F.; Goyens, P. Effects of thirty elements on bone metabolism. J. Trace Elem. Med. Biol. 2015, 32, 86-106. [CrossRef]

59. Merkiel, S.; Chalcarz, W. Dietary intake in 6-year-old children from southern Poland. Part 2-Vitamin and mineral intakes. BMC Pediatrics 2014, 14, 310. [CrossRef]

60. Pettifor, J.M. Calcium and vitamin D metabolism in children in developing countries. Ann. Nutr. Metab. 2014, 64, 15-22. [CrossRef]

61. Merkiel, S.; Chalcarz, W. Analysis of mineral intake in preschool children from Turek. Med. Rodz. 2016, 1, 7-13.

62. Zhu, K.; Prince, R.L. Calcium and bone. Clin. Biochem. 2012, 45, 936-942. [CrossRef] [PubMed]

63. Strnad, M. Sol. Salt and cancer. Acta Med. Croatica. 2010, 64, 159-161.

64. Hu, J.; La Vecchia, C.; Morrison, H.; Negri, E.; Mery, L. Canadian Cancer Registries Epidemiology Research Group. Salt processed meat and the risk of cancer. Eur. J. Cancer Prev. 2011, 20, 132-139. [CrossRef]

65. Huybrechts, I.; De Henauw, S. Energy and nutrient intakes by pre-school children in Flanders-Belgium. Br. J. Nutr. 2007, 98, 600-610. [CrossRef] [PubMed]

66. Huybrechts, I.; Maes, L.; Vereecken, C.; De Keyzer, W.; De Bacquer, D. High dietary supplements intakes among Flemish preschools. Appetite 2010, 54, 340-345. [CrossRef]

67. Wielgos, B.; Leszczyńska, T.; Kopeć, A.; Cieślik, E.; Piątkowska, E.; Pysz, M. Assesment of intake of minerals with daily diets by children aged 10-12 years from Malopolska region. Rocz. Panstw. Zakl. Hig. 2012, 63, 329-337.

68. Domellöf, M.; Thorsdottir, I.; Thorstensen, K. Health effects of different dietary iron intakes: A systematic literature review for the 5th Nordic Nutrition Recommendations. Food Nutr. Res. 2013, 57, 21667. [CrossRef]

69. Singh, M. Role of micronutrients for physical growth and mental development. Indian J. Pediatr. 2004, 71, 59-62. [CrossRef]

70. Tsai, C.J.; Leitzmann, M.F.; Willett, W.C.; Giovannucci, E.L. Heme and non-heme iron consumption and risk of gallstone disease in men. Am. J. Clin. Nutr. 2007, 85, 518-522. [CrossRef]

71. Ndayisaba, A.; Kaindlstorfer, C.; Wenning, G.K. Iron in Neurodegeneration-Cause or Consequence? Front. Neurosci. 2019, 13, 180. [CrossRef] [PubMed]

72. Zhang, W.; Iso, H.; Ohira, T.; Date, C.; Tanabe, N.; Kikuchi, S.; Tamakoshi, A. JACC Study Group. Associations of dietary iron intake with mortality from cardiovascular disease. J. Epidemiol. 2012, 22, 484-493. [CrossRef] [PubMed]

73. Sadowska, J.; Krzymuska, A. The estimation of complementation of the nursery school food rations by parents of pre-school children. Bromat. Chem. Toksykol. 2010, 52, 203-211.

74. Malhotra, K.; Baggott, P.J.; Livingstone, J. Vitamin D in the foot and ankle-A review of the literature. J. Am. Podiatr. Med. Assoc. 2020, 110, 1-11. [CrossRef] [PubMed] 